Homology, Homotopy and Applications, vol.20(2), 2018, pp.259-280

\title{
ON POLYHEDRAL PRODUCT SPACES OVER POLYHEDRAL JOINS
}

\author{
ELIZABETH VIDAURRE
}

(communicated by Charles A. Weibel)

\begin{abstract}
The construction of a simplicial complex given by polyhedral joins (introduced by Anton Ayzenberg), generalizes Bahri, Bendersky, Cohen and Gitler's $J$-construction and simplicial wedge construction. This article gives a cohomological decomposition of a polyhedral product over a polyhedral join. A formula for the Hilbert-Poincaré series is given, which generalizes Ayzenberg's formula for the moment-angle complex.
\end{abstract}

\section{Introduction}

From a simplicial complex and pairs of topological spaces, polyhedral product spaces give a family of spaces (see Definition 2.2). The spaces that have the form of a polyhedral product include moment-angle complexes, complements of complex coordinate subspace arrangements, and intersections of quadrics among others. In certain cases, polyhedral products provide geometric realizations of right-angled Artin groups and the Stanley-Reisner ring.

One can define the polyhedral join, $Z_{K}^{*}(\underline{X}, \underline{A})$, similarly to the polyhedral product by replacing the cartesian product with the topological or simplicial join [1]. When the pairs $(\underline{X}, \underline{A})$ are simplicial complexes $(\underline{L}, \underline{K})$, the associated polyhedral join is a simplicial complex. A natural question that arises concerning a polyhedral product over such a simplicial complex is: what is the cohomology of the polyhedral product space over a polyhedral join, $Z_{Z_{K}^{*}(\underline{L}, \underline{K})}(\underline{X}, \underline{A})$, in terms of the simplicial complexes $K$, $K_{i}, L_{i}$ and the cohomology of the spaces $(\underline{X}, \underline{A})$ ? In this paper, we answer this question and, in the case of simplicial pairs $\left(L_{i}, K_{i}\right)$ given by a simplex and a subsimplicial complex, analyze the product in the cohomology ring.

Polyhedral joins behave well with polyhedral products. For example, there is the following homeomorphism between real moment-angle complexes and moment-angle complexes [3]:

$$
Z_{Z_{K}^{*}\left(\Delta^{1}, \partial \Delta^{1}\right)}\left(D^{1}, S^{0}\right)=Z_{K}\left(D^{2}, S^{1}\right) .
$$

This means that any moment-angle complex can be expressed as a real moment-angle complex. This equivalence has since been used by many $[11,6,9,10,8]$.

Received September 29, 2017, revised March 7, 2018; published on June 13, 2018.

2010 Mathematics Subject Classification: 05E45, 13F55, $14 \mathrm{~F} 45$.

Key words and phrases: Cohomology ring, moment-angle complex, simplicial complex, StanleyReisner ring, simplicial wedge construction.

Article available at http://dx.doi.org/10.4310/HHA.2018.v20.n2.a13

Copyright (C) 2018, International Press. Permission to copy for private use granted. 
Using polyhedral joins $Z_{K}^{*}(\underline{L}, \underline{K})$, this can be generalized to arbitrary polyhedral products. It allows for a give and take in dimension. A polyhedral product can be expressed as another polyhedral product of either lower dimensional spaces $(\underline{X}, \underline{A})$ over a higher dimensional simplicial complex, or of higher dimensional spaces $(\underline{X}, \underline{A})$ over a lower dimensional simplicial complex.

We use a spectral sequence constructed by Bahri, Bendersky, Cohen and Gitler that converges to the cohomology of $Z_{K}(\underline{X}, \underline{A})$ in terms of the long exact sequence for the pairs $\left(X_{i}, A_{i}\right)$. It gives a Kunneth-like formula for the general polyhedral product. In Section 3 we show how the spectral sequence reduces the problem to analyzing the map $H^{*}\left(\Sigma l k_{L_{i}}(\sigma)\right) \rightarrow H^{*}\left(\Sigma l k_{K_{i}}(\sigma)\right)$ induced by the inclusion of links $l k_{K_{i}}(\sigma) \hookrightarrow l k_{L_{i}}(\sigma)$, where $\sigma$ is a simplex in $K_{i}$. Since there is no convenient way to describe the induced map in full generality, we consider two cases of pairs of simplicial complexes.

In Section 4, we restrict to the pairs $\left(L_{i}, K_{i}\right)=\left(\Delta^{l_{i}}, K_{i}\right)$ where $\Delta^{l_{i}}$ is the $l_{i^{-}}$ simplex. With this pair, the associated polyhedral join is called the composed simplicial complex $K\left(K_{1}, \ldots, K_{m}\right)$. This pair covers the case of the homeomorphism between moment-angle complexes and real moment-angle complexes. As a consequence of Theorem 4.1, we give the corresponding Hilbert-Poincaré series. For the polyhedral product $Z_{K\left(K_{1}, \ldots, K_{m}\right)}(\underline{C A}, \underline{A})$, we define a multigraded structure and give the multigraded Betti numbers in Proposition 4.8. Moreover, the ring structure of $H^{*}\left(Z_{Z_{K}(\underline{L}, \underline{K})}(\underline{X}, \underline{A})\right)$ is analyzed. This amounts to a description of the StanleyReisner ideal of $K\left(K_{1}, \ldots, K_{m}\right)$, given in Proposition 4.9.

Qibing Zheng also studies the pair $\left(\Delta^{l_{i}}, K_{i}\right)$ in [12] to derive an Alexander duality isomorphism for certain classes of polyhedral products. Our methods are distinct from his and are used for a different purpose.

Finally, in Section 5, we study the case given by the pair $\left(L_{i}, K_{i}\right)=\left(L_{i}, \emptyset\right)$ as the map induced by the inclusion of links can be fully described. The cohomology groups will be given in Theorem 5.1.

\section{Acknowledgments}

This work is part of the author's doctoral dissertation at the City University of New York Graduate Center. The author would like to thank Martin Bendersky for his guidance throughout this research.

\section{Polyhedral product spaces}

Let $[m]=\{1,2, \ldots, m\}$ denote the set of integers from 1 to $m$. An abstract simplicial complex, $K$, on $[m]$ is a subset of the power set of $[m]$, such that:

1. $\emptyset \in K$.

2. If $\sigma \in K$ with $\tau \subset \sigma$, then $\tau \in K$.

An $n$-simplex is the full power set of $[n+1]$ and is denoted $\Delta^{n}$. Associated to an abstract simplicial complex is its geometric realization, denoted $\mathcal{K}$ or $|K|$ (also called a geometric simplicial complex). A (geometric) $n$-simplex, $\Delta^{n}$, is the convex hull of $n+1$ points. 
We do not assume $m$ is minimal, i.e. there may exist $[n] \subsetneq[m]$ such that $K$ is contained in the power set of $[n]$. In particular, we allow ghost vertices $\{i\} \subset[m]$ such that $\{i\} \notin K$.

Let $K$ and $L$ be simplicial complexes on sets $[m]$ and $[n]$, respectively. A map from $[m]$ to $[n]$ induces a simplicial map from $K$ to $L$ if it satisfies the property that simplices map to simplices.

Definition 2.1. Let $I$ be a subset of $[m]$. The full subcomplex of $K$ in $I$ is denoted $K_{I}$. It is a simplicial complex on the set $I$ and defined by

$$
K_{I}:=\{\sigma \in K \mid \sigma \subset I\}
$$

It will sometimes be helpful to use the notation $\left.K\right|_{I}$ and it is often called the restriction of $K$ to $I$ in the literature.

Given an abstract simplicial complex $K$, let $\mathcal{S}_{K}$ be the category with simplices of $K$ as the objects and inclusions as the morphisms. In particular, for $\sigma, \tau \in o b\left(\mathcal{S}_{K}\right)$, there is a morphism $\sigma \rightarrow \tau$ whenever $\sigma \subset \tau$. Define $\mathcal{C W}$ to be the category of CWcomplexes and continuous maps. Define $(\underline{X}, \underline{A})$ to be a collection of pairs of $\mathrm{CW}$ complexes $\left\{\left(X_{i}, A_{i}\right)\right\}_{i=1}^{m}$, where $A_{i}$ is a subspace of $X_{i}$ for all $i$.

Definition 2.2. Given an abstract simplicial complex $K$ on $[m]$, simplices $\sigma, \tau$ of $K$ and a collection of pairs of $C W$-complexes $(\underline{X}, \underline{A})$, define a diagram $D: \mathcal{S}_{K} \rightarrow \mathcal{C W}$ given by

$$
D(\sigma)=\prod_{i \in[m]} Y_{i}, \quad \text { where } Y_{i}= \begin{cases}X_{i} & i \in \sigma, \\ A_{i} & i \in[m] \backslash \sigma .\end{cases}
$$

For a morphism $f: \sigma \rightarrow \tau$, the functor $D$ maps $f$ to $\iota: D(\sigma) \rightarrow D(\tau)$ where $\iota$ is the canonical injection.

The polyhedral product space, $Z_{K}(\underline{X}, \underline{A}) \subset \prod_{i \in[m]} X_{i}$ is

$$
Z_{K}(\underline{X}, \underline{A}):=\underset{\sigma \in K}{\operatorname{colim}} D(\sigma)=\bigcup_{\sigma \in K} D(\sigma) .
$$

Notice that it suffices to take the colimit over the maximal simplices of $K$. In fact, simplicial complexes can be defined by their maximal simplices and this description will be used throughout. In the case where $\left(X_{i}, A_{i}\right)=(X, A)$ for all $i$, we write $Z_{K}(X, A)$.

Some examples of polyhedral products are moment-angle complexes $Z_{K}\left(D^{2}, S^{1}\right)$, which have the homotopy type of the complement of a complex coordinate subspace arrangement, and Davis-Januszkiewicz spaces $Z_{K}\left(C P^{\infty}, p t\right)$, which have a StanleyReisner ring as cohomology ring. For a simple example, consider the following.

Example 1. Let $K$ be the boundary of a 2-simplex.

$$
\begin{aligned}
Z_{K}\left(D^{1}, S^{0}\right) & =D^{1} \times D^{1} \times S^{0} \cup D^{1} \times S^{0} \times D^{1} \cup S^{0} \times D^{1} \times D^{1} \\
& =\partial\left(D^{1} \times D^{1} \times D^{1}\right) \\
& \cong S^{2}
\end{aligned}
$$

In general, $Z_{\partial \Delta^{m}}\left(D^{1}, S^{0}\right) \cong S^{m}$ (see examples in [2]). 
Next we will define the polyhedral smash product, a space analogous to the polyhedral product with the smash product operation in place of the cartesian product. Define $\mathcal{C W}_{*}$ to be the category of based $\mathrm{CW}$-complexes and based continuous maps.

Definition 2.3. Let the CW-pairs $(\underline{X}, \underline{A})$ be pointed. Likewise, define a functor $\widehat{D}(\sigma): \mathcal{S}_{K} \rightarrow \mathcal{C} \mathcal{W}_{*}$ by

$$
\widehat{D}(\sigma)=\wedge Y_{i}, \quad \text { where } Y_{i}= \begin{cases}X_{i} & i \in \sigma, \\ A_{i} & i \notin \sigma .\end{cases}
$$

Then the polyhedral smash product is

$$
\widehat{Z}_{K}(\underline{X}, \underline{A})=\bigcup \widehat{D}(\sigma) .
$$

The following theorem of Bahri, Bendersky, Cohen and Gitler (BBCG) gives a decomposition of a suspension of a polyhedral product.

Theorem 2.4 (Splitting Theorem, [2]). Let $\left(\underline{X_{I}}, \underline{A_{I}}\right)=\left\{\left(X_{i}, A_{i}\right)\right\}_{i \in I}$. Then

$$
\Sigma Z_{K}(\underline{X}, \underline{A}) \simeq \Sigma\left(\bigvee_{I \subset[m]} \widehat{Z}_{K_{I}}\left(\underline{X_{I}}, \underline{A_{I}}\right)\right),
$$

where $\Sigma$ denotes the reduced suspension.

Given any simplicial complex, the following procedure allows for the construction of an infinite family of associated simplicial complexes. Let $\mathcal{S C}$ be the category with simplicial complexes as the objects and simplicial maps as the morphisms.

Definition 2.5 (Ayzenberg [1]). Let $K$ be a simplicial complex on $m$ vertices and $\sigma$ a simplex of $K$. Let $(\underline{L}, \underline{K})=\left\{L_{i}, K_{i}\right\}_{i \in[m]}$ be $m$ pairs of simplicial complexes, where $K_{i}$ is a subsimplicial complex of $L_{i}$ and both are defined on the index set $\left[l_{i}\right]$. Consider a functor $D^{*}: \mathcal{S}_{K} \rightarrow \mathcal{S C}$ defined in the following way

$$
D^{*}(\sigma)=\underset{i \in[m]}{*} Y_{i}, \text { where } Y_{i}= \begin{cases}L_{i} & i \in \sigma, \\ K_{i} & i \notin \sigma .\end{cases}
$$

The associated polyhedral join is the colimit of the diagram

$$
Z_{K}^{*}(\underline{L}, \underline{K}):=\underset{\sigma \in K}{\operatorname{colim}} D^{*}(\sigma)=\bigcup_{\sigma \in K} D^{*}(\sigma) .
$$

Note that $Z_{K}^{*}(\underline{L}, \underline{K})$ is a subsimplicial complex of $\underset{i \in[m]}{*} L_{i}$, which is a simplicial complex on the set $\left[\sum_{i \in[m]} l_{i}\right]$. In particular, $D^{*}(\sigma)$ is the join of simplicial complexes $L_{i}$ for $i \in \sigma$ and simplicial complexes $K_{j}$ for $j \in[m] \backslash \sigma$.

Definition 2.6. Let $K$ be a simplicial complex on the set $[m]$ and $\left\{L_{i}\right\}_{i \in[m]}$ be simplicial complexes on the sets $\left[l_{i}\right]$.

The composition of $K$ with $\left\{L_{i}\right\}_{i=1}^{m}$, denoted $K\left(L_{1}, \ldots, L_{m}\right)$, is defined to be

$$
K\left(L_{1}, \ldots, L_{m}\right):=Z_{K}^{*}\left(\Delta^{l_{i}-1}, L_{i}\right) .
$$


The composition $K\left(L_{1}, \ldots, L_{m}\right)$ may also be defined by the following condition: for subsets $\sigma_{i} \subset\left[l_{i}\right]$, the set $\sigma=\sigma_{1} \sqcup \cdots \sqcup \sigma_{m}$ is a simplex of $K\left(L_{1}, \ldots, L_{m}\right)$ whenever the set $\left\{i \in[m] \mid \sigma_{i} \notin L_{i}\right\}$ is a simplex of $K$.

It is noteworthy that simplicial complexes form an operad where the simplicial complex on $m$ vertices is viewed as an $m$-adic operation. See [1] for more details.

The composition is a generalization of the J-construction [3] and the simplicial wedge construction [7].

The link of $\sigma \in K$, denoted $l k_{K}(\sigma)$, is a simplicial complex on the set $[m] \backslash \sigma$ defined by $\tau \in l k_{K}(\sigma)$ if and only if $\sigma \cup \tau \in K$. This indexing set is used to be consistent with the definition in [1]. Given the indexing set of this complex, the link may have ghost vertices that are not ghost vertices of $K$.

Example 2. This is an example of a composition of simplicial complexes $K\left(L_{1}\right.$, $\left.\ldots, L_{m}\right)$. Let $m=3$ and $K=\{\{1\},\{2,3\}\}$ :

$$
\begin{aligned}
& l_{1}=1 \quad \text { and } \quad L_{1}=\{\emptyset\}, \\
& l_{2}=1 \quad \text { and } \quad L_{2}=\{\{21\}\}, \\
& l_{3}=2 \quad \text { and } \quad L_{3}=\{\{31\},\{32\}\},
\end{aligned}
$$

where $L_{1}$ consists of a ghost vertex, $L_{2}$ a single vertex, and $L_{3}$ two vertices. Then

$$
\begin{array}{rlrlr}
K\left(L_{1}, L_{2}, L_{3}\right) & = & D^{*}(\{2,3\}) & \cup & D^{*}(\{1\}) \\
& = & L_{1} * \Delta^{0} * \Delta^{1} & \cup & \Delta^{0} * L_{2} * L_{3} \\
& =\{\emptyset\} *\{21\} *\{31,32\} \cup\{11\} *\{21\} *\{31\},\{32\} \\
& = & \{21,31,32\}, & & \{11,21,31\},\{11,21,32\} .
\end{array}
$$

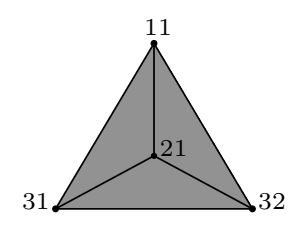

Figure 1: Example of a composed simplicial complex.

Composed simplicial complexes have a nice relationship with polyhedral products. Note that in the following proposition the indexing set of $(\underline{X}, \underline{A})$ is different than it has been up to this point, and so it will be explicitly labeled. The notation used will be $Z_{K}\left(X_{i}, A_{i}\right)_{i \in I}$ for some indexing set $I$.

Proposition 2.7 (Ayzenberg [1, Proposition 5.1]). Let $K$ be a simplicial complex on $m$ vertices and $\left\{L_{i}\right\}_{i \in[m]}$ be simplicial complexes with $l_{i}$ vertices. We have $\sum_{i \in[m]} l_{i}$ pairs $\left(X_{i j}, A_{i j}\right)$ with $i \in[m]$ and $j \in\left[l_{i}\right]$. Then

$$
Z_{K\left(L_{1}, \ldots, L_{m}\right)}\left(X_{i j}, A_{i j}\right)_{i \in[m], j \in\left[l_{i}\right]}=Z_{K}\left(\prod_{j \in\left[l_{i}\right]} X_{i j}, Z_{L_{i}}\left(X_{i j}, A_{i j}\right)_{j \in\left[l_{i}\right]}\right)_{i \in[m]} .
$$

These spaces are equal, not just homeomorphic. The proof involves a shuffling of the spaces $X_{i}$ and $A_{i}$ as in the proof of Proposition 2.8. 
Using methods of [1] we prove the analogous result for the polyhedral smash product.

Proposition 2.8. Let $K$ be a simplicial complex on $m$ vertices and $\left\{L_{i}\right\}_{i \in[m]}$ be simplicial complexes with $l_{i}$ vertices. Then

$$
\widehat{Z}_{K\left(L_{1}, \ldots, L_{m}\right)}\left(X_{i j}, A_{i j}\right)_{i \in[m], j \in\left[l_{i}\right]}=\widehat{Z}_{K}\left(\bigwedge_{j \in\left[l_{i}\right]} X_{i j}, \widehat{Z}_{L_{i}}\left(X_{i j}, A_{i j}\right)_{j \in\left[l_{i}\right]}\right)_{i \in[m]} .
$$

Proof. Suppose we have a simplex $\sigma \in K$ and a simplex $\tau_{i} \in L_{i}$ for each $1 \leqslant i \leqslant m$. Then

$$
\begin{aligned}
\widehat{Z}_{K}\left(\bigwedge_{j \in\left[l_{i}\right]} X_{i j}, \widehat{Z}_{L_{i}}\left(X_{i j}, A_{i j}\right)_{j \in\left[l_{i}\right]}\right)_{i \in[m]} \\
=\bigcup_{\sigma}\left(\bigwedge_{i \in \sigma}\left(\bigwedge_{j \in\left[l_{i}\right]} X_{i j}\right) \wedge \bigwedge_{i \in[m] \backslash \sigma}\left(\bigcup_{\tau_{i}}\left(\bigwedge_{j \in \tau_{i}} X_{i j}\right) \wedge\left(\bigwedge_{j \in\left[l_{i}\right] \backslash \tau_{i}} A_{i j}\right)\right)\right) \\
\left.=\bigcup_{\sigma, \tau_{i}}\left(\bigwedge_{i \in \sigma}\left(\bigwedge_{j \in\left[l_{i}\right]} X_{i j}\right) \wedge \bigwedge_{i \in[m] \backslash \sigma}\left(\bigwedge_{j \in \tau_{i}} X_{i j}\right) \wedge\left(\bigwedge_{j \in\left[l_{i}\right] \backslash \tau_{i}} A_{i j}\right)\right)\right) .
\end{aligned}
$$

Since simplices in $\sigma^{\prime} \in K\left(L_{1}, \ldots, L_{m}\right)$ are of the form $\sigma^{\prime}=\left(\bigcup_{i \in[m] \backslash \sigma} \tau_{i}\right) \cup\left(\bigcup_{i \in \sigma} \Delta^{l_{i}-1}\right)$,

$$
\bigwedge_{i \in \sigma}\left(\bigwedge_{j \in\left[l_{i}\right]} X_{i j}\right) \wedge \bigwedge_{i \in[m] \backslash \sigma}\left(\bigwedge_{j \in \tau_{i}} X_{i j}\right)=\bigwedge_{i j \in \sigma^{\prime}} X_{i j}
$$

Note that $\bigcup_{i \in[m] \backslash \sigma}\left[l_{i}\right] \backslash \tau_{i}=\left[\Sigma l_{i}\right] \backslash \sigma^{\prime}$, and thus

$$
\bigwedge_{i \in[m] \backslash \sigma}\left(\bigwedge_{j \in\left[l_{i}\right] \backslash \tau_{i}} A_{i j}\right)=\bigwedge_{i j \in\left[\Sigma l_{i}\right] \backslash \sigma^{\prime}} A_{i j}
$$

And finally,

$$
\bigcup_{\sigma^{\prime}}\left(\bigwedge_{i j \in \sigma^{\prime}} X_{i j} \wedge \bigwedge_{i j \in\left[\Sigma l_{i}\right] \backslash \sigma^{\prime}} A_{i j}\right)=\widehat{Z}_{K\left(L_{1}, \ldots, L_{m}\right)}\left(X_{i j}, A_{i j}\right)_{i \in[m], j \in\left[l_{i}\right]}
$$

Similarly, we can make the same type of argument for the polyhedral join in place of the composed simplicial complex for the most general form.

Theorem 2.9. Given $m$ pairs of simplicial complexes $(\underline{L}, \underline{K})$ where $L_{i}$ and $K_{i}$ are simplicial complexes on the vertex set $\left[l_{i}\right]$ ( $L_{i}$ may have ghost vertices). Taking $\sum_{i \in[m]} l_{i}$ pairs $\left(X_{i j}, A_{i j}\right)$, we have

$$
Z_{Z_{K}^{*}(\underline{L}, \underline{K})}\left(X_{i j}, A_{i j}\right)_{i \in[m], j \in\left[l_{i}\right]}=Z_{K}\left(Z_{L_{i}}\left(X_{i j}, A_{i j}\right)_{j \in\left[l_{i}\right]}, Z_{K_{i}}\left(X_{i j}, A_{i j}\right)_{j \in\left[l_{i}\right]}\right)_{i \in[m]} .
$$

Proof. The proof is similar to Proposition 2.8. However, a simplex $\sigma^{\prime} \in Z_{K}^{*}(\underline{L}, \underline{K})$ is of the form

$$
\sigma^{\prime}=\left(\bigcup_{i \in[m] \backslash \sigma} \tau_{i}\right) \cup\left(\bigcup_{i \in \sigma} \rho_{i}\right),
$$

where $\sigma \in K, \tau_{i} \in K_{i}$ and $\rho_{i} \in L_{i}$. 
The complexes $K_{i}$ must have an indexing set of the same cardinality of the indexing set of $L_{i}$; otherwise, the statement is not true. In particular, $L_{i}$ may have ghost vertices. Keep in mind that including ghost vertices does change the polyhedral product by multiplying by $A_{i}$ where $i$ is a ghost vertex.

In [3], Bahri, Bendersky, Cohen and Gitler show that a moment-angle complex can be expressed as a real moment-angle complex. Since $Z_{\partial \Delta^{m}}\left(D^{1}, S^{0}\right) \cong S^{m}$ (see Example 1), we have the following as a consequence of Theorem 2.9.

Corollary 2.10 (Bahri, Bendersky, Cohen and Gitler [3]). Real moment-angle complexes over a composed simplicial complex are homeomorphic to moment-angle complexes:

$$
Z_{K\left(\partial \Delta_{1}, \ldots, \partial \Delta_{1}\right)}\left(D^{1}, S^{0}\right) \cong Z_{K}\left(D^{2}, S^{1}\right)
$$

\subsection{The BBCG spectral sequence}

Recall that our goal is to compute the cohomology of $Z_{Z_{K}^{*}(\underline{L}, \underline{K})}(\underline{X}, \underline{A})$ in terms of $K, K_{i}, L_{i}, H^{*}\left(X_{i j}\right)$ and $H^{*}\left(A_{i j}\right)$. To do so, we will use a spectral sequence developed by BBCG [4]. It gives a Kunneth-like formula for the cohomology of a polyhedral product as long as the pairs $(\underline{X}, \underline{A})$ satisfy the following freeness condition.

Definition 2.11. Given the pair $\left(X_{i}, A_{i}\right)$, the associated long exact sequence is given by

$$
\cdots \stackrel{\delta}{\rightarrow} \widetilde{H}^{*}\left(X_{i} / A_{i}\right) \stackrel{g}{\rightarrow} H^{*}\left(X_{i}\right) \stackrel{f}{\rightarrow} H^{*}\left(A_{i}\right) \stackrel{\delta}{\rightarrow} \widetilde{H}^{*+1}\left(X_{i} / A_{i}\right) \stackrel{g}{\rightarrow} \cdots
$$

Assume that the cohomology groups of the pair have the following decomposition:

$$
\begin{aligned}
H^{*}\left(A_{i}\right) & =B_{i} \oplus E_{i}, \\
H^{*}\left(X_{i}\right) & =B_{i} \oplus C_{i}, \\
\widetilde{H}^{*}\left(X_{i} / A_{i}\right) & =C_{i} \oplus W_{i},
\end{aligned}
$$

where $W_{i}$ is $s E_{i}$, the suspension of $E_{i}$. Additionally, assume $1 \in B_{i}$, and for $b \in B_{i}$, $c \in C_{i}, e \in E_{i}, w \in W_{i}=s E_{i}$, we have

$$
b \stackrel{f}{\mapsto} b \stackrel{\delta}{\mapsto} 0, \quad c \stackrel{g}{\mapsto} c \stackrel{f}{\mapsto} 0, \quad e \stackrel{\delta}{\mapsto} w \stackrel{g}{\mapsto} 0 .
$$

Before defining the spectral sequence, we will give some notation and recall the definition of a half smash product:

1. for $\sigma=\left\{i_{1}, \ldots, i_{k}\right\}$, define $\widehat{X}^{\sigma}:=X_{i_{1}} \wedge \cdots \wedge X_{i_{k}}$ and $A^{\sigma}=A_{i_{1}} \times \cdots \times A_{i_{k}}$,

2. the complement of a set $\sigma \subset[m]$ is $\sigma^{c}=[m] \backslash \sigma$,

3. given a basepoint $x_{0} \in X$, the right half smash product $X \rtimes Y=(X \times Y) /$ $\left(x_{0} \times Y\right)$,

4. for a subset $I$ and a simplex $\sigma$ such that $\sigma \subset I$, define

$$
Y^{I, \sigma}:=\bigotimes_{i \in \sigma} C_{i} \otimes \bigotimes_{i \in I-\sigma} B_{i}
$$

Choosing a lexicographical ordering for the simplices of $K$ gives a filtration of the associated polyhedral product space and polyhedral smash product, which in turn leads to a spectral sequence converging to the reduced cohomology of $Z_{K}(\underline{X}, \underline{A})$ and a spectral sequence converging to the reduced cohomology of $\widehat{Z}_{K}(\underline{X}, \underline{A})$. The $E_{1}^{s, t}$ term for $Z_{K}(\underline{X}, \underline{A})$ has the following description. 
Theorem 2.12 (Bahri, Bendersky, Cohen and Gitler [4]). There exist spectral sequences

$$
\begin{aligned}
& E_{r}^{s, t} \rightarrow H^{*}\left(Z_{K}(\underline{X}, \underline{A})\right), \\
& \widehat{E}_{r}^{s, t} \rightarrow H^{*}\left(\widehat{Z}_{K}(X, A)\right),
\end{aligned}
$$

with $E_{1}^{s, t}=\widetilde{H}^{t}\left((\widehat{X / A})^{\sigma} \rtimes A^{\sigma^{c}}\right)$ and $\widehat{E}_{1}^{s, t}=\widetilde{H}^{t}\left((\widehat{X / A})^{\sigma} \wedge \widehat{A}^{\sigma^{c}}\right)$ where $s$ is index of $\sigma$ in the lexicographical ordering and the differential $d_{r}: E_{r}^{s, t} \rightarrow E_{r}^{s+r, t+1}$ is induced by the coboundary map $\delta: E \rightarrow W=s E$. Moreover, the spectral sequence is natural for embeddings of simplicial maps with the same number of vertices and with respect to maps of pairs. The natural quotient map

$$
Z_{K}(\underline{X}, \underline{A}) \rightarrow \widehat{Z}_{K}(\underline{X}, \underline{A})
$$

induces a morphism of spectral sequences and the splitting theorem (2.4) induces a morphism of spectral sequences.

Following [4], Definition 2.11 and the Künneth theorem imply that the entries $\widetilde{H}^{t}\left((\widehat{X / A})^{\sigma} \wedge \widehat{A}^{\sigma^{c}}\right)$ in the first page of the spectral sequence for $\widehat{Z}_{K}(\underline{X}, \underline{A})$ decompose as a direct sum of spaces $W^{N} \otimes C^{S} \otimes B^{T} \otimes E^{J}$ such that $N \cup S=\bar{\sigma}, T \cup J=\sigma^{c}$ and $N, S, J, T$ are disjoint. We have that $S$ is a simplex in $K$ as $N \cup S$ is a simplex in $K$. Since the differential is induced by the coboundary $\delta: E \rightarrow W$, consider all the possible summands $W^{N} \otimes C^{S} \otimes B^{T} \otimes E^{J}$ for $S$ and $T$ are fixed. It must be the case that $N$ is a simplex in $K$ and that $N$ is a subset of $[m] \backslash(S \cup T)$. Therefore all such $N$ correspond to simplices in the link of $S$ in $K$ restricted to the vertex set $[m] \backslash(S \cup T)$.

Theorem 2.13 (Bahri, Bendersky, Cohen and Gitler [4]). Let $(\underline{X}, \underline{A})$ satisfy the decomposition described in Definition 2.11

$$
\begin{aligned}
& H^{*}\left(A_{i}\right)=B_{i} \oplus E_{i}, \\
& H^{*}\left(X_{i}\right)=B_{i} \oplus C_{i} .
\end{aligned}
$$

Then

$$
H^{*}\left(Z_{K}(\underline{X}, \underline{A})\right)=\bigoplus_{I \subset[m], \sigma \subset I} E^{I^{c}} \otimes Y^{I, \sigma} \otimes \widetilde{H}^{*}\left(\Sigma\left|l k(\sigma)_{I^{c}}\right|\right)
$$

where:

1. $\sigma$ is a simplex in $K$,

2. $l k(\sigma)_{I^{c}}=\{\tau \subset[m] \backslash I \mid \tau \cup \sigma \in K\}$ is the link of $\sigma$ in $K$ restricted to the set $[m] \backslash I$,

3. $Y^{I, \sigma}=\bigotimes_{i \in \sigma} C_{i} \otimes \bigotimes_{i \in I-\sigma} B_{i}$, and

4. $\widetilde{H}^{*}(\Sigma \emptyset)=1$.

Theorem 2.14 (Bahri, Bendersky, Cohen and Gitler [4]). Let

$$
\begin{aligned}
\widetilde{H}^{*}\left(A_{i}\right) & =\widetilde{B}_{i} \oplus E_{i}, \\
\widetilde{H}^{*}\left(X_{i}\right) & =\widetilde{B}_{i} \oplus C_{i} .
\end{aligned}
$$

Then

$$
H^{*}\left(\widehat{Z}_{K}(\underline{X}, \underline{A})\right)=\bigoplus_{I \subset[m], \sigma \subset I} E^{I^{c}} \otimes Y^{I, \sigma} \otimes \widetilde{H}^{*}\left(\Sigma\left|l k(\sigma)_{I^{c}}\right|\right)
$$


where:

1. $\sigma$ is a simplex in $K$,

2. $l k(\sigma)_{I^{c}}=\{\tau \subset[m] \backslash I \mid \tau \cup \sigma \in K\}$ is the link of $\sigma$ in $K$ restricted to the set $[m] \backslash I$,

3. $Y^{I, \sigma}=\bigotimes_{i \in \sigma} C_{i} \otimes \bigotimes_{i \in I-\sigma} \widetilde{B}_{i}$ where $\widetilde{B}_{i}=B_{i} \backslash\{1\}$,

4. $\widetilde{H}^{*}(\Sigma \emptyset)=1$.

Consequently, assuming that the cohomology of the pairs $\left(X_{i j}, A_{i j}\right)$ satisfy the freeness condition, then once the kernel, image and cokernel of the pair $\left(Z_{L_{i}}(\underline{X}, \underline{A})\right.$, $\left.Z_{K_{i}}(\underline{X}, \underline{A})\right)$ are computed, this theorem can be applied.

\section{The general polyhedral join}

We ultimately aim to understand the cohomology of the space $Z_{Z_{K}^{*}(\underline{L}, \underline{K})}(\underline{X}, \underline{A})$. By Theorem 5.1, this is equivalent to computing the cohomology of the space

$$
Z_{K}\left(Z_{L_{i}}\left(X_{i j}, A_{i j}\right)_{j \in\left[l_{i}\right]}, Z_{K_{i}}\left(X_{i j}, A_{i j}\right)_{j \in\left[l_{i}\right]}\right)_{i \in[m]} .
$$

As a starting point, we apply Theorem 2.13.

Fix an $i$. The inclusion $K_{i} \hookrightarrow L_{i}$ induces the obvious inclusion $Z_{K_{i}}(\underline{X}, \underline{A}) \hookrightarrow$ $Z_{L_{i}}(\underline{X}, \underline{A})$, which in turn induces a map in cohomology

$$
H^{*}\left(Z_{L_{i}}(\underline{X}, \underline{A})\right) \stackrel{\phi}{\rightarrow} H^{*}\left(Z_{K_{i}}(\underline{X}, \underline{A})\right) .
$$

By Theorem 2.13, we have that

$$
\begin{aligned}
H^{*}\left(Z_{L_{i}}(\underline{X}, \underline{A})\right)= & \bigoplus_{\substack{\sigma \in L_{i} \\
\sigma \subset I}} E^{I^{c}} \otimes Y^{I, \sigma} \otimes \widetilde{H}^{*}\left(\Sigma\left|l k_{L_{i}}(\sigma)_{I^{c}}\right|\right) \\
= & \left(\bigoplus_{\substack{\tau \in K_{i} \\
\tau \subset I}} E^{I^{c}} \otimes Y^{I, \tau} \otimes \widetilde{H}^{*}\left(\Sigma\left|l k_{L_{i}}(\tau)_{I^{c}}\right|\right)\right) \\
& \oplus\left(\bigoplus_{\substack{\sigma \notin K_{i} \\
\sigma \subset I}} E^{I^{c}} \otimes Y^{I, \sigma} \otimes \widetilde{H}^{*}\left(\Sigma\left|l k_{L_{i}}(\sigma)_{I^{c}}\right|\right)\right)
\end{aligned}
$$

and

$$
H^{*}\left(Z_{K_{i}}(\underline{X}, \underline{A})\right)=\bigoplus_{\tau \in K_{i}, \tau \subset I} E^{I^{c}} \otimes Y^{I, \tau} \otimes \widetilde{H}^{*}\left(\Sigma\left|l k_{K_{i}}(\tau)_{I^{c}}\right|\right)
$$

where $l k_{L_{i}}(\sigma)_{I^{c}}$ is the link of $\sigma$ in $L_{i}$ restricted to the vertex set $\left[l_{i}\right] \backslash I$. Therefore, understanding the map $\phi$ is reduced to finding the image of the factor $\alpha \in$ $\widetilde{H}^{*}\left(\Sigma\left|l k_{L_{i}}(\tau)_{I^{c}}\right|\right)$ for $\tau \in K_{i}$. Recall (from the discussion before Theorem 2.13) that $\alpha$ corresponds to the exponent of the $W \mathrm{~s}$ in a summand $E^{J} \otimes W^{N} \otimes C^{\tau} \otimes B^{I \backslash \tau}$ of

$$
\widetilde{H}^{*}\left(\widehat{X / A}^{(N \cup \tau)}\right) \otimes \widetilde{H}^{*}\left(\widehat{A}^{(N \cup \tau)^{c}}\right)
$$

in the $E_{1}$ page of the BBCG spectral sequence. If $N \cup \tau$ is a simplex in $K_{i}$, then $\widetilde{H}^{*}\left(\widehat{X / A}^{(N \cup \tau)}\right) \otimes \widetilde{H}^{*}\left(\widehat{A}^{(N \cup \tau)^{c}}\right)$ in the spectral sequence for $Z_{K_{i}}(\underline{X}, \underline{A})$ maps to 
$\widetilde{H}^{*}\left(\widehat{X / A}^{(N \cup \tau)}\right) \otimes \widetilde{H}^{*}\left(\widehat{A}^{(N \cup \tau)^{c}}\right)$ in the spectral sequence of $Z_{L_{i}}(\underline{X}, \underline{A})$ by the naturality of the spectral sequence for embeddings of simplicial maps. In particular, if $N \cup \tau \in K_{i}$, then

$$
\phi: E^{J} \otimes W^{N} \otimes C^{\tau} \otimes B^{I \backslash \tau} \mapsto E^{J} \otimes W^{N} \otimes C^{\tau} \otimes B^{I \backslash \tau}
$$

and if $N \cup \tau \notin K_{i}$, then

$$
\phi: E^{J} \otimes W^{N} \otimes C^{\tau} \otimes B^{I \backslash \tau} \mapsto 0 .
$$

This is the dual of the inclusion

$$
l k_{K_{i}}(\sigma)_{I^{c}} \hookrightarrow l k_{L_{i}}(\sigma)_{I^{c}} .
$$

In other words, the map $\phi$ is induced by the inclusion $l k_{K_{i}}(\sigma)_{I^{c}} \hookrightarrow l k_{L_{i}}(\sigma)_{I^{c}}$.

\section{Composed simplicial complexes}

Recall that a composed simplicial complex is the polyhedral join $Z_{K}^{*}\left(\Delta^{l_{i}-1}, L_{i}\right)$, denoted $K\left(L_{1}, \ldots, L_{m}\right)$, which is of particular importance because it yields the equivalence between moment-angle complexes and some real moment-angle complexes. In this case, the link of any simplex in $\Delta^{l_{i}-1}$ is a simplex, and hence the geometric realization of the link is contractible. This means the cohomology of the suspension of the link is trivial, and therefore so is a summand $E^{I^{c}} \otimes Y^{I, \sigma} \otimes \widetilde{H}^{*}\left(\Sigma\left|l k(\sigma)_{I^{c}}\right|\right)$ unless $I=[m]$. In particular, the image, kernel and cokernel of the map

$$
H^{*}\left(\prod_{j \in\left[l_{i}\right]} X_{i j}\right) \rightarrow H^{*}\left(Z_{L_{i}}\left(X_{i j}, A_{i j}\right)\right)
$$

can be computed. The following proposition gives the cohomology of the polyhedral product over a composed simplicial complex in terms of the cohomology of the pairs and the simplicial complexes (using notation described after Definition 2.11).

Theorem 4.1. Given $m$ simplicial complexes $\left\{L_{i}\right\}_{i \in[m]}$, where $L_{i}$ is a complex on the set $\left[l_{i}\right]$. For each $i \in[m]$, let there be a family of pairs $\left(X_{i j}, A_{i j}\right)_{j \in\left[l_{i}\right]}$ satisfying Definition 2.11

$$
\begin{aligned}
& H^{*}\left(A_{i j}\right)=B_{i j} \oplus E_{i j}, \\
& H^{*}\left(X_{i j}\right)=B_{i j} \oplus C_{i j} .
\end{aligned}
$$

Then

$$
\begin{aligned}
& H^{*}\left(Z_{K\left(L_{1}, \ldots, L_{m}\right)}(X, A)\right)=\bigoplus_{\substack{I, \sigma, J \\
\tau, \rho, \rho^{\prime}}} \widetilde{H}^{*}\left(\Sigma\left|l k(\sigma)_{I^{c}}\right|\right) \\
& \otimes\left(\bigotimes_{k \in[m] \backslash I} E^{J^{c}} \otimes \widetilde{H}^{*}\left(\Sigma\left|l k(\tau)_{J^{c}}\right|\right) \otimes Y^{J, \tau}\right) \\
& \otimes\left(\bigotimes_{s \in \sigma} Y^{\left[l_{s}\right], \rho^{\prime}} \otimes \bigotimes_{s^{\prime} \in I \backslash \sigma} Y^{\left[l_{s^{\prime}}\right], \rho}\right),
\end{aligned}
$$

where:

1. $I \subset[m]$ and $\sigma$ is a simplex in $K$, with $\sigma \subset I$, 
2. $J \subsetneq\left[l_{k}\right]$ and $\tau$ is a simplex in $L_{k}$, with $\tau \subset J$,

3. $\rho^{\prime}$ is a nonsimplex in $L_{s}$ and $\rho$ is a simplex in $L_{s^{\prime}}$.

Proof. Recall Ayzenberg's Theorem,

$$
Z_{K\left(L_{1}, \ldots, L_{m}\right)}\left(X_{i j}, A_{i j}\right)=Z_{K}\left(\prod_{j \in\left[L_{i}\right]} X_{i j}, Z_{L_{i}}\left(X_{i j}, A_{i j}\right)\right)
$$

and that we want to find the kernel, $C_{i}$, image, $B_{i}$, and cokernel, $E_{i}$, of the induced $\operatorname{map} H^{*}\left(\prod_{j \in\left[l_{i}\right]} X_{i j}\right) \rightarrow H^{*}\left(Z_{L_{i}}\left(X_{i j}, A_{i j}\right)\right)$.

Since

$$
\begin{aligned}
H^{*}\left(\prod_{j=1}^{l_{i}} X_{i j}\right) & =\bigotimes_{j=1}^{l_{i}}\left(B_{i j} \oplus C_{i j}\right) \\
& =\bigoplus_{\rho \in \Delta^{l_{i}-1}} Y^{\left[l_{i}\right], \rho} \\
& =\bigoplus_{\rho \in L_{i}} Y^{\left[l_{i}\right], \rho} \oplus \bigoplus_{\rho^{\prime} \notin L_{i}} Y^{\left[l_{i}\right], \rho^{\prime}}
\end{aligned}
$$

and, by Theorem 2.13,

$$
H^{*}\left(Z_{L_{i}}\left(X_{i j}, A_{i j}\right)_{j \in\left[l_{i}\right]}\right)=\bigoplus_{\rho \in L_{i}} Y^{\left[l_{i}\right], \rho} \oplus \bigoplus_{J \subsetneq\left[l_{i}\right], \tau \subset J} E^{\left[l_{i}\right]-J} \otimes \widetilde{H}^{*}\left(\Sigma\left|l k(\tau)_{J^{c}}\right|\right) \otimes Y^{J, \tau}
$$

we have

$$
\begin{aligned}
B_{i} & =\bigoplus_{\rho \in L_{i}} Y^{\left[l_{i}\right], \rho}, \\
C_{i} & =\bigoplus_{\rho^{\prime} \notin L_{i}} Y^{\left[l_{i}\right], \rho^{\prime}}, \\
E_{i} & =\bigoplus_{\tau \in L_{i}, \tau \subset J \subsetneq\left[l_{i}\right]} E^{\left[l_{i}\right]-J} \otimes \widetilde{H}^{*}\left(\Sigma\left|l k(\tau)_{J^{c}}\right|\right) \otimes Y^{J, \tau} .
\end{aligned}
$$

Notice that $C_{i}$ is the Stanley-Reisner ideal of $L_{i}, I\left(L_{i}\right)$, and $B_{i}$ is the StanleyReisner ring of $L_{i}, S R\left(L_{i}\right)$ (see Corollary 4.3). Now applying Theorem 2.13 again,

$$
H^{*}\left(Z_{K}\left(\prod_{j \in\left[l_{i}\right]} X_{i j}, Z_{L_{i}}\left(X_{i j}, A_{i j}\right)\right)\right)=\bigoplus_{I \subset[m], \sigma \subset I} E^{I^{c}} \otimes Y^{I, \sigma} \otimes \widetilde{H}^{*}\left(\Sigma\left|l k(\sigma)_{I^{c}}\right|\right) .
$$

Substituting (4.3) and expanding,

$$
\begin{aligned}
E^{[m]-I} & =\bigotimes_{k \in[m]-I} E_{k} \\
& =\bigotimes_{k \in[m]-I} \bigoplus_{J, \tau} E^{\left[l_{k}\right]-J} \otimes \widetilde{H}^{*}\left(\Sigma\left|l k(\tau)_{J^{c}}\right|\right) \otimes Y^{J, \tau} \\
& =\bigoplus_{J, \tau}\left(\bigotimes_{k \in[m]-I} E^{\left[l_{k}\right]-J} \otimes \widetilde{H}^{*}\left(\Sigma\left|l k(\tau)_{J^{c}}\right|\right) \otimes Y^{J, \tau}\right) .
\end{aligned}
$$


Next, substituting (4.1) and (4.2) and expanding,

$$
\begin{aligned}
Y^{I, \sigma} & =\bigotimes_{s \in \sigma} C_{s} \otimes \bigotimes_{s^{\prime} \in I-\sigma} B_{s^{\prime}} \\
& =\bigotimes_{s \in \sigma}\left(\bigoplus_{\rho^{\prime} \notin L_{s}} Y^{\left[l_{s}\right], \rho^{\prime}}\right) \otimes \bigotimes_{s^{\prime} \in I-\sigma}\left(\bigoplus_{\rho \in L_{s^{\prime}}} Y^{\left[l_{s^{\prime}}\right], \rho}\right) \\
& =\bigoplus_{\rho^{\prime} \notin L_{s}}\left(\bigotimes_{s \in \sigma} Y^{\left[l_{s}\right], \rho^{\prime}}\right) \otimes \bigoplus_{\rho \in L_{s^{\prime}}}\left(\bigotimes_{s^{\prime} \in I-\sigma} Y^{\left[l_{s^{\prime}}\right], \rho}\right) \\
& =\bigoplus_{\rho^{\prime}, \rho}\left(\bigotimes_{s \in \sigma} Y^{\left[l_{s}\right], \rho^{\prime}} \otimes \bigotimes_{s^{\prime} \in I-\sigma} Y^{\left[l_{s^{\prime}}\right], \rho}\right) .
\end{aligned}
$$

The proposition follows.

Recall that the decomposition of $H^{*}\left(Z_{K}(\underline{X}, \underline{A})\right)$ in Theorem 2.12 differs from the decomposition for $H^{*}\left(\widehat{Z}_{K}(\underline{X}, \underline{A})\right)$ by the presence of $1 \in B_{i j}$. As a consequence, the same proof provides a decomposition for $H^{*}\left(\widehat{Z}_{K\left(L_{1}, \ldots, L_{m}\right)}(\underline{X}, \underline{A})\right)$. Below is an example of how Proposition 4.1 can be used to compute the Poincaré series for the cohomology of a polyhedral product over a composed simplicial complex.

Example 3. Suppose we have that $K$ is two ghost vertices, $L_{1}$ is a point and $L_{2}$ is two points. Namely, $K=\{\emptyset\}$ is indexed by $[2], L_{1}=\{\{11\}\}, L_{2}=\{\{21\},\{22\}\}$. Given a pair of spaces such that $\widetilde{H}^{*}(X)=\left\langle b_{4}, c_{6}\right\rangle$ and $\widetilde{H}^{*}(A)=\left\langle e_{2}, b_{4}\right\rangle$, we will compute the Poincaré series for $H^{*}\left(\widehat{Z}_{K\left(L_{1}, L_{2}\right)}(X, A)\right)$.

1. For $I=\emptyset$, the only possible simplex, $\sigma$, is the empty set. Since $J_{1} \neq[11]$, the only choice for $J_{1}$ and $\tau_{1}$ is the empty set, then $l k\left(\tau_{1}\right)_{J_{1}^{c}}=L_{1}$ is contractible. There is no contribution to the Poincaré series. This means we will only consider $I$ such that $1 \in I$.

2. In the case $I=\{1\}$ and $\sigma=\emptyset$, we consider subsets, $J_{k}$, of $L_{k}$ for $k \in[m] \backslash I$, and simplices $\rho_{s}^{\prime} \in L_{s}$ and $\rho_{s^{\prime}} \in L_{s^{\prime}}$ for $s \in \sigma$ and $s^{\prime} \in I \backslash \sigma$ :

(a) For $J_{2}=\emptyset$ and $\tau_{2}=\emptyset, l k\left(\tau_{2}\right)_{J_{2}^{c}}=L_{2}$ and $\widetilde{H}^{*}\left(\Sigma\left|l k\left(\tau_{2}\right)_{J_{2}^{c}}\right|\right)=\left\langle\iota_{1}\right\rangle$ :

i. If $\rho_{1}=\emptyset$, then $\bar{P}\left(E_{21} \otimes E_{22} \otimes \iota_{1} \otimes B_{11}\right)=t^{9}$ is contributed to the Poincaré series;

ii. If $\rho_{1}=\{11\}$, then $\bar{P}\left(E_{21} \otimes E_{22} \otimes \iota_{1} \otimes C_{11}\right)=t^{11}$ is contributed.

(b) Similarly, with $J_{2}=\{21\}$ and $\tau_{2}=\emptyset, l k\left(\tau_{2}\right)_{J_{2}^{c}}=\{\{22\}\}$ is contractible.

(c) If $J_{2}=\{21\}$ and $\tau_{2}=\{21\}$, then $l k\left(\tau_{2}\right)_{J_{2}^{c}}=\emptyset$ :

i. If $\rho_{1}=\emptyset$, then $\bar{P}\left(C_{21} \otimes E_{22} \otimes 1 \otimes B_{11}\right)=t^{12}$;

ii. If $\rho_{1}=\{11\}$, then $\bar{P}\left(C_{21} \otimes E_{22} \otimes 1 \otimes C_{11}\right)=t^{14}$.

(d) If $J_{2}=\{22\}, \tau_{2}=\{22\}$ then $l k\left(\tau_{2}\right)_{J_{2}^{c}}=\emptyset$ :

i. If $\rho_{1}=\emptyset$, then $\bar{P}\left(C_{22} \otimes E_{21} \otimes 1 \otimes B_{11}\right)=t^{12}$;

ii. If $\rho_{1}=\{11\}$, then $\bar{P}\left(C_{22} \otimes E_{21} \otimes 1 \otimes C_{11}\right)=t^{14}$.

3. For $I=\{2,1\}$ and $\sigma=\emptyset, l k(\sigma)_{I}=\emptyset$ :

(a) For $\rho_{1}=\emptyset$ : 
i. If $\rho_{2}=\emptyset$, then $\bar{P}\left(B_{11} \otimes B_{21} \otimes B_{22}\right)=t^{12}$;

ii. If $\rho_{2}=\{21\}$, then $\bar{P}\left(B_{11} \otimes C_{21} \otimes B_{22}\right)=t^{14}$;

iii. If $\rho_{2}=\{22\}$, then $\bar{P}\left(B_{11} \otimes B_{21} \otimes C_{22}\right)=t^{14}$.

(b) For $\rho_{1}=\{11\}$ :

i. If $\rho_{2}=\emptyset$, then $\bar{P}\left(C_{11} \otimes B_{21} \otimes B_{22}\right)=t^{14}$;

ii. If $\rho_{2}=\{21\}$, then $\bar{P}\left(C_{11} \otimes C_{21} \otimes B_{22}\right)=t^{16}$;

iii. If $\rho_{2}=\{22\}$, then $\bar{P}\left(C_{11} \otimes B_{21} \otimes C_{22}\right)=t^{16}$.

In conclusion,

$$
\bar{P}\left(H^{*}\left(\widehat{Z}_{K\left(L_{1}, L_{2}\right)}(X, A)\right)\right)=t^{9}+t^{11}+3 t^{12}+5 t^{14}+2 t^{16} .
$$

Since

$$
\begin{aligned}
K\left(L_{1}, L_{2}\right) & =L_{1} * L_{2} \\
& =\{\{11,21\},\{11,22\}\}
\end{aligned}
$$

a simplicial complex with three vertices and two edges, we can see that this is consistent with Example 5.8 in [4]. Their example computes the Poincaré series for the polyhedral product over a simplicial complex with three vertices and two edges, and spaces with equivalent cohomology. We obtained the same answer using a different method.

Definition 4.2. Let $K$ be a simplicial complex on $m$ vertices and $k$ be a ring. Consider $k[m]:=k\left[v_{1}, \ldots, v_{m}\right]$, the ring of polynomials in $m$ indeterminates. The generalized Stanley-Reisner ideal of $K, I(K)$, is generated by square-free monomials indexed by the nonsimplices of $K$

$$
I(K)=\left\langle v_{i_{1}} \cdots v_{i_{n}} \mid\left\{i_{1}, \ldots, i_{n}\right\} \notin K\right\rangle .
$$

The Stanley-Reisner (or face) ring of a simplicial complex, $K$, is denoted $k[K]$ and is defined as

$$
k[K]=k[m] / I(K) \cdot
$$

The following is another version of Theorem 4.1 which highlights the role of the Stanley-Reisner ring (see Definition 4.2).

Corollary 4.3. Following Definition 2.11, suppose we have a decomposition

$$
\begin{aligned}
& H^{*}\left(A_{i j}\right)=B_{i j} \oplus E_{i j}, \\
& H^{*}\left(X_{i j}\right)=B_{i j} \oplus C_{i j},
\end{aligned}
$$

with $E_{i j}$ the cokernel of $H^{*}\left(X_{i j}\right) \rightarrow H^{*}\left(A_{i j}\right), B_{i j}$ the image, and $C_{i j}$ the kernel. Then

$$
\begin{aligned}
H^{*}\left(Z_{K\left(L_{1}, \ldots, L_{m}\right)}(X, A)\right)= & \bigoplus_{I, \sigma, J, \tau} \widetilde{H}^{*}\left(\Sigma\left|l k(\sigma)_{I^{c}}\right|\right) \\
& \otimes\left(\bigotimes_{k \in[m] \backslash I}\left(E^{\left[l_{k}\right]-J} \otimes \widetilde{H}^{*}\left(\Sigma\left|l k(\tau)_{J^{c}}\right|\right)\right) \otimes Y^{J, \tau}\right) \\
& \otimes\left(\bigotimes_{s \in \sigma} I\left(L_{s}\right) \otimes \bigotimes_{s^{\prime} \in I \backslash \sigma} S R\left(L_{s^{\prime}}\right)\right),
\end{aligned}
$$

where 
1. $I \subset[m]$ and $\sigma$ is a simplex in $K$, with $\sigma \subset I$,

2. $J \subsetneq\left[l_{k}\right] . \tau$ is a simplex in $L_{k}$, with $\tau \subset J$,

3. $\rho^{\prime}$ is a nonsimplex in $L_{s}$ and $\rho$ is a simplex in $L_{s^{\prime}}$.

Corollary 4.4. Let $I^{\prime}=I_{1}^{\prime} \sqcup \cdots \sqcup I_{m}^{\prime} \subset\left[\Sigma l_{i}\right]$ and $\sigma^{\prime}=\sigma_{1}^{\prime} \sqcup \cdots \sqcup \sigma_{m}^{\prime}$ a simplex in $K\left(L_{1}, \ldots, L_{m}\right)$ with $\sigma^{\prime} \subset\left[\sum l_{i}\right] \backslash I^{\prime}$. Then

$$
\widetilde{H}^{*}\left(\Sigma\left|l k\left(\sigma^{\prime}\right)_{I^{\prime}}\right|\right)=\bigotimes_{k \in I} \widetilde{H}^{*}\left(\Sigma\left|l k\left(\sigma_{k}^{\prime}\right)_{I_{k}^{\prime}}\right|\right) \otimes \widetilde{H}^{*}\left(\Sigma\left|l k(\sigma)_{I}\right|\right),
$$

where $I=\left\{i \in[m] \mid I_{i}^{\prime} \neq \emptyset\right\}$ and $\sigma=\left\{i \in[m] \backslash I \mid \sigma_{i}^{\prime} \notin L_{i}\right\}$.

Proof. By Theorem 2.13,

$$
H^{*}\left(Z_{K\left(L_{1}, \ldots, L_{m}\right)}(X, A)\right)=\bigoplus_{\sigma^{\prime}, I^{\prime}} E^{\left[\sum l_{i}\right]-I^{\prime}} \otimes \widetilde{H}^{*}\left(\Sigma\left|l k\left(\sigma^{\prime}\right)_{I^{\prime c}}\right|\right) \otimes Y^{I^{\prime}, \sigma^{\prime}} .
$$

Moreover, by Proposition 4.1, we have that $E^{\left[\sum l_{i}\right]-I^{\prime}}=\bigotimes_{k \in[m]-I} E^{\left[l_{k}\right]-J_{k}}$ whenever $I_{i}^{\prime}=\left[l_{i}\right]$ for all $i \in I$ and $I_{k}^{\prime}=J_{k}$ for all $k \in[m]-I$. We also have that

$$
Y^{I^{\prime}, \sigma^{\prime}}=\bigotimes_{k \in[m]-I} Y^{J_{k}, \tau} \otimes\left(\bigotimes_{s \in \sigma} Y^{\left[l_{s}\right], \rho^{\prime}}\right) \otimes\left(\bigotimes_{s^{\prime} \in I-\sigma} Y^{\left[l_{s^{\prime}}\right], \rho}\right),
$$

whenever $\sigma^{\prime}=(\cup \tau) \cup\left(\cup \rho^{\prime}\right) \cup(\cup \rho)$. Thus for $k \in[m]-I, \sigma_{k}^{\prime}=\tau$. Similarly, for $s \in$ $\sigma, \sigma_{s}^{\prime}=\rho^{\prime} \notin L_{s}$. In other words $s \in \sigma$ if and only if $\sigma_{s}^{\prime} \notin L_{s}$. Lastly, for $s^{\prime} \in I-\sigma$, $\sigma_{s^{\prime}}^{\prime}=\rho$. A change of notation is used so that the proposition is not stated in terms of complements of sets.

Example 4. Refer to Example 2 of $K\left(L_{1}, L_{2}, L_{3}\right)$ for details of the construction. We will find $\widetilde{H}^{*}\left(\Sigma\left|l k_{K\left(L_{1}, L_{2}, L_{3}\right)}\left(\sigma^{\prime}\right)_{I^{\prime}}\right|\right)$ in terms of the links in $K, L_{1}, L_{2}, L_{3}$ for several cases of $\sigma^{\prime} \in K\left(L_{1}, L_{2}, L_{3}\right)$ and $I^{\prime} \subset\left[\sum l_{i}\right]$.

1. Suppose $\sigma^{\prime}=\{32\}$. Then $\sigma_{1}^{\prime}=\emptyset, \sigma_{2}^{\prime}=\emptyset, \sigma_{3}^{\prime}=\{32\}$.

(a) If $I^{\prime}=\{11,31\}$, then $I_{1}^{\prime}=\{11\}, I_{2}^{\prime}=\emptyset, I_{3}^{\prime}=\{31\}$. This means $I=\{1,3\}$ and $\sigma=\emptyset$. Thus

$$
\begin{aligned}
\widetilde{H}^{*}\left(\Sigma\left|l k\left(\sigma^{\prime}\right)_{I^{\prime}}\right|\right) & =\widetilde{H}^{*}\left(\Sigma\left|l k\left(\sigma_{1}^{\prime}\right)_{I_{1}^{\prime}}\right|\right) \otimes \widetilde{H}^{*}\left(\Sigma\left|l k\left(\sigma_{3}^{\prime}\right)_{I_{3}^{\prime}}\right|\right) \otimes \widetilde{H}^{*}\left(\Sigma\left|l k(\sigma)_{I}\right|\right) \\
& =\widetilde{H}^{*}(\Sigma \emptyset) \otimes \widetilde{H}^{*}(\Sigma \emptyset) \otimes \widetilde{H}^{*}(\Sigma|\{\{1\},\{3\}\}|) \\
& =1 \otimes 1 \otimes \widetilde{H}^{*}\left(S^{1}\right) .
\end{aligned}
$$

This is consistent with $\left|l k\left(\sigma^{\prime}\right)_{I^{\prime}}\right|=\left|\{\{11,21\},\{21,31\}\}_{I^{\prime}}\right|=|\{\{11\},\{31\}\}| \simeq$ $S^{0}$.

(b) If $I^{\prime}=\{31,21\}$, then $I=\{2,3\}$ and $\sigma=\emptyset$. Thus $\widetilde{H}^{*}\left(\Sigma\left|l k\left(\sigma^{\prime}\right)_{I^{\prime}}\right|\right)=1 \otimes 1 \otimes 0$. This is consistent with $\left|l k\left(\sigma^{\prime}\right)_{I^{\prime}}\right|=|\{\{31,21\}\}|=\Delta^{1}$, which is contractible.

2. Suppose $\sigma^{\prime}=\{11,32\}$ and $I^{\prime}=\{31\}$. Then $I=\{3\}$ and $\sigma=\{1\}$. Moreover, $\widetilde{H}^{*}\left(\Sigma\left|l k\left(\sigma^{\prime}\right)_{I^{\prime}}\right|\right)=1 \otimes 1$, which is consistent with $l k\left(\sigma^{\prime}\right)_{I^{\prime}}=\emptyset$.

Recall that Definition 2.1 of the full subcomplex, $K_{I}$ or $\left.K\right|_{I}$, and that the notation $\mathcal{K}$ or $|K|$ denotes the geometric realization of $K$. Specializing Proposition 4.1 to the case $(\underline{X}, \underline{A})=(\underline{C A}, \underline{A})$ we have the following corollary. 


\section{Corollary 4.5.}

$H^{*}\left(Z_{K\left(L_{1}, \ldots, L_{m}\right)}(\underline{C A}, \underline{A})\right)=\bigoplus_{\substack{I \subset[m], J_{k} \subset\left[l_{k}\right], J_{k} \neq \emptyset}} \widetilde{H}^{*}\left(\Sigma \mathcal{K}_{I}\right) \otimes\left(\bigotimes_{k \in I} \widetilde{H}^{*}\left(\left.\Sigma \mathcal{L}_{k}\right|_{J_{k}}\right) \otimes \widetilde{H}^{*}\left(\widehat{A}^{J_{k}}\right)\right)$, where $\widehat{A}^{J}:=\bigwedge_{j \in[J]} A_{j}$.

Proof. Recall $L_{k}$ is a simplicial complex on the set $\left[l_{k}\right]$. For the pair $\left(C A_{i}, A_{i}\right), B_{i}=1$, $C_{i}=0$ and $E_{i}=\widetilde{H}^{*}\left(A_{i}\right)$. We have that $Y^{J, \tau} \neq 0$ whenever $\tau=\emptyset$, so that $l k\left(\tau_{k}\right)_{J_{k}}=$ $\left.L_{k}\right|_{J_{k}}$. Also, if $\sigma \neq \emptyset$, then $Y^{\left[l_{i}\right], \rho^{\prime}}=0$ because the emptyset is not a nonsimplex of any simplicial complex. Since $\sigma=\emptyset$, it follows that $l k(\sigma)_{I^{c}}=K_{I^{c}}$. Recall that $J_{k} \neq\left[l_{k}\right]$ and hence $J_{k}^{c} \neq \emptyset$.

An immediate corollary of Corollary 4.5 is a computation of the Poincaré series of

$$
\widetilde{H}^{*}\left(Z_{K\left(L_{1}, \ldots, L_{m}\right)}(\underline{C A}, \underline{A})\right) \text {. }
$$

\section{Corollary 4.6.}

$$
\bar{P}\left(\widetilde{H}^{*}\left(Z_{K\left(L_{1}, \ldots, L_{m}\right)}(\underline{C A}, \underline{A})\right)\right)=\sum_{\substack{I \subset[m], J_{k} \subset\left[l_{k}\right], J_{k} \neq \emptyset}} \bar{P}\left(\widetilde{H}^{*}\left(\Sigma \mathcal{K}_{I}\right)\right) \prod_{k \in I} \bar{P}\left(\widetilde{H}^{*}\left(\left.\Sigma \mathcal{L}_{k}\right|_{J_{k}}\right)\right) \bar{P}\left(\widetilde{H}^{*}\left(\widehat{A}^{J_{k}}\right)\right) .
$$

Remark 4.1. It follows from the splitting theorem (2.4) that the Poincaré series can also be written in the following form:

$$
\bar{P}\left(\widetilde{H}^{*}\left(Z_{K\left(L_{1}, \ldots, L_{m}\right)}(\underline{C A}, \underline{A})\right)\right)=\sum_{B \subset[m]} \bar{P}\left(\widetilde{H}^{*}\left(\Sigma \mathcal{K}_{B}\right)\right) \prod_{b \in B} \bar{P}\left(\widetilde{H}^{*}\left(Z_{L_{b}}(\underline{C A}, \underline{A})\right)\right) .
$$

This generalizes the computation for $\left(D^{2}, S^{1}\right)$ in Ayzenberg to the case $(\underline{C A}, \underline{A})$. The above formulas can be proven instead by also using Ayzenberg's result on the homotopy type of the composition, which will be described in further detail in the following proof.

Alternate proof of Corollary 4.6. Ayzenberg's Lemma 7.5 states that for $J=$ $\cup J_{i} \subset\left[\sum l_{i}\right], K\left(L_{1}, \ldots, L_{m}\right)_{J}=K_{B}\left(\left.L_{b_{1}}\right|_{J_{b_{1}}}, \ldots,\left.L_{b_{k}}\right|_{J_{b_{k}}}\right)$ where $B=\left\{b_{i} \mid J_{i} \neq \emptyset\right\}$ and Corollary 6.2 states that $\left|K\left(L_{1}, \ldots, L_{m}\right)\right| \simeq|K| *\left|L_{1}\right| * \ldots *\left|L_{m}\right|$, and hence $\left|K\left(L_{1}, \ldots, L_{m}\right)\right| \simeq|K| \wedge \Sigma\left|L_{1}\right| \wedge \cdots \wedge \Sigma\left|L_{m}\right|$. Now, using the splitting theorem (2.4), and the wedge lemma, which states that $\widehat{Z}_{K}(\underline{X}, \underline{A}) \simeq \Sigma|K| \wedge \widehat{A}^{[m]}$, we have the following:

$$
\begin{aligned}
\widetilde{H}^{*}\left(Z_{K\left(L_{1}, \ldots, L_{m}\right)}(\underline{C A}, \underline{A})\right)=\bigoplus_{J} \widetilde{H}^{*}\left(\Sigma\left|K\left(L_{1}, \ldots, L_{m}\right)_{J}\right| \wedge \widehat{A}^{J}\right) \\
=\bigoplus_{B, J_{b_{1}}, \ldots, J_{b_{k}}} \widetilde{H}^{*}\left(\Sigma\left|K_{B}\left(\left.L_{b_{1}}\right|_{J_{b_{1}}}, \ldots, L_{b_{k}} \mid J_{b_{k}}\right)\right| \wedge \widehat{A}^{J_{b_{1}}} \wedge \cdots \wedge \widehat{A}^{J_{b_{k}}}\right) \\
=\bigoplus_{B, J_{b_{1}}, \ldots, J_{b_{k}}} \widetilde{H}^{*}\left(\Sigma\left|K_{B}\right| \wedge \Sigma\left|\left(L_{b_{1}} \mid J_{b_{1}}\right)\right| \wedge \cdots \wedge \Sigma\left|\left(L_{b_{k}} \mid J_{b_{k}}\right)\right| \wedge \widehat{A}^{J_{b_{1}}} \wedge \cdots \wedge \widehat{A}^{J_{b_{k}}}\right) \\
=\bigoplus_{B, J_{b_{1}}, \ldots, J_{b_{k}}} \widetilde{H}^{*}\left(\Sigma\left|K_{B}\right|\right) \otimes \bigotimes_{b_{i} \in B} \widetilde{H}^{*}\left(\Sigma\left|\left(L_{b_{i}} \mid J_{b_{i}}\right)\right| \wedge \widehat{A}^{J_{b_{i}}}\right)
\end{aligned}
$$




$$
\begin{aligned}
& =\bigoplus_{B} \widetilde{H}^{*}\left(\Sigma\left|K_{B}\right|\right) \otimes \bigotimes_{b_{i} \in B} \bigoplus_{J_{b_{i}}} \widetilde{H}^{*}\left(\Sigma\left|\left(L_{b_{i}} \mid J_{b_{i}}\right)\right| \wedge \widehat{A}^{J_{b_{i}}}\right) \\
& =\bigoplus_{B} \widetilde{H}^{*}\left(\Sigma\left|K_{B}\right|\right) \otimes \bigotimes_{b_{i} \in B} \widetilde{H}^{*}\left(Z_{L_{b_{i}}}(\underline{C A}, \underline{A})\right) .
\end{aligned}
$$

\subsection{Multigraded Betti numbers}

Let $\mathbb{k}$ be the ground field and $\mathbb{k}[m]=\mathbb{k}\left[v_{1}, \ldots, v_{m}\right]$ be the ring of polynomials in $m$ indeterminates. The ring $\mathbb{k}[m]$ has a $\mathbb{Z}^{m}$-grading defined by $\operatorname{deg}\left(v_{i}\right)=(0, \ldots, 2, \ldots, 0)$ with 2 in the $i$-th place. Given a free resolution $\cdots \rightarrow R^{-i} \rightarrow \cdots \rightarrow \mathbb{k}[K]$ by $\mathbb{Z}^{m_{-}}$ graded $\mathbb{k}[m]$ modules, we have the Tor-module

$$
\operatorname{Tor}_{\mathbb{k}[m]}(\mathbb{k}[K], \mathbb{k})=\bigoplus_{i \in \mathbb{Z} \geqslant 0, \bar{j} \in \mathbb{Z}^{m}} \operatorname{Tor}_{\mathbb{k}[m]}^{-i, 2 \bar{j}}(\mathbb{k}[K], \mathbb{k}) .
$$

The multigraded Betti numbers of a simplicial complex are then defined in terms of the multigraded structure of the Tor-module. The $(-i, 2 \bar{j})$-th Betti number of $K$ is the dimension of $\operatorname{Tor}_{\mathbb{k}[m]}^{-i, 2 \bar{j}}(\mathbb{k}[K], \mathbb{k})$ over $\mathbb{k}$ :

$$
\beta_{\mathbb{k}}^{-i, 2 \bar{j}}(K):=\operatorname{dim}_{\mathbb{k}}\left(\operatorname{Tor}_{\mathbb{k}[m]}^{-i, 2 \bar{j}}(\mathbb{k}[K], \mathbb{k})\right) .
$$

Since the cohomology ring of the moment-angle complex is $\operatorname{Tor}_{\mathbb{k}[m]}(\mathbb{k}[K], \mathbb{k})$, the multigraded Betti numbers of a simplicial complex have a topological interpretation in terms of $Z_{K}\left(D^{2}, S^{1}\right)[5]$. We adapt this interpretation for an arbitrary polyhedral product of pairs $(\underline{C A}, \underline{A})$.

Moreover, taking into account Hochster's theorem:

$$
\beta_{\mathbb{k}}^{-i, 2 \bar{j}}(K)=\operatorname{dim} \tilde{H}^{|J|-i-1}\left(K_{J} ; \mathbb{k}\right),
$$

where there is a straightforward association between $\bar{j} \in \mathbb{Z}^{m}$ and a subset $J \subset[m]$. Then using the splitting theorem (2.4) and wedge lemma we give an analogous definition for the multigraded Betti numbers of a polyhedral product.

Definition 4.7. For $i \in \mathbb{Z}$ and $J \subset[m]$, the multigraded Betti numbers of $Z_{K}(\underline{C A}, \underline{A})$, denoted $\beta^{i, J}\left(Z_{K}(\underline{C A}, \underline{A})\right)$, are defined as

$$
\beta^{i, J}\left(Z_{K}(\underline{C A}, \underline{A})\right):=\operatorname{dim} \widetilde{H}^{i}\left(\Sigma \mathcal{K}_{J} \wedge \widehat{A}^{J}\right) .
$$

Let $s$ and $t_{1}, \ldots, t_{m}$ be indeterminates such that $\bar{t}^{J}=t_{1}^{j_{1}} \cdots t_{m}^{j_{m}}$ where $j_{i}=1$ if $i \in J$ and $j_{i}=0$ otherwise. The beta-polynomial of $Z_{K}(\underline{C A}, \underline{A})$ is defined as

$$
\beta_{Z_{K}(\underline{C A}, \underline{A})}(s, \bar{t}):=\sum_{i \in \mathbb{Z}, J \subset[m]} \beta^{i, J}\left(Z_{K}(\underline{C A}, \underline{A})\right) s^{i} \bar{t}^{J}
$$

and the reduced beta-polynomial

$$
\widetilde{\beta}_{Z_{K}(\underline{C A}, \underline{A})}(s, \bar{t}):=\beta_{Z_{K}(\underline{C A}, \underline{A})}(s, \bar{t})-1=\sum_{i \in \mathbb{Z}, \emptyset \neq J \subset[m]} \beta^{i, J}\left(Z_{K}(\underline{C A}, \underline{A})\right) s^{i} \bar{t}^{J} .
$$

The definition of multigraded Betti numbers of a simplicial complex is given in terms of a Tor algebra and the Stanley-Reisner ring, which are in terms of indeterminates in degree 2 . When considering the $(-i, 2 j)$-th Betti number of $K$ and applying 
Hochster's theorem, the $(-i, 2 j)$-th Betti number should be the dimension of the cohomology of $K_{J}$ in degree $(-i+2 j)-j-1=-i+j-1$ where $|J|=j$. This is equivalent to Definition 4.7 since $\widetilde{H}^{i}\left(\Sigma \mathcal{K}_{J} \wedge \widehat{A}^{J}\right)=\widetilde{H}^{i-j-1}\left(K_{J}\right)$, with a change of variables for the shift in cohomological degree.

Proposition 4.8. The beta-polynomial of $Z_{K\left(L_{1}, \ldots, L_{m}\right)}(\underline{C A}, \underline{A})$ can be expressed in terms of the beta-polynomial of the polyhedral products associated to each of the simplicial complexes $K, L_{1}, \ldots, L_{m}$ :

$$
\beta_{Z_{K\left(L_{1}, \ldots L_{m}\right)}(\underline{C A}, \underline{A})}(s, \bar{t})=\beta_{Z_{K}\left(D^{1}, S^{0}\right)}\left(s, \widetilde{\beta}_{Z_{L_{1}}(\underline{C A}, \underline{A})}(s, \bar{t}), \ldots, \widetilde{\beta}_{Z_{L_{m}}(\underline{C A}, \underline{A})}(s, \bar{t})\right) .
$$

Proof. Let $i^{\prime} \in \mathbb{Z}, J=\cup J_{i} \subset\left[\sum l_{i}\right], B=\left\{b_{i} \in[m] \mid J_{i} \neq \emptyset\right\}=\left\{b_{1}, \ldots, b_{k}\right\}, n+p=$ $i^{\prime}, r+\sum r_{s}=n, \sum c_{s}=p$. Using Corollary 4.5, we have

$$
\begin{aligned}
& \beta_{Z_{K\left(L_{1}, \ldots, L_{m}\right)}(\underline{C A}, \underline{A})}(s, \bar{t})=\sum_{i^{\prime}, J} \operatorname{dim} \widetilde{H}^{i^{\prime}}\left(\Sigma K\left(L_{1}, \ldots, L_{m}\right)_{J} \wedge \widehat{A}^{J}\right) s^{i^{\prime}} \bar{t}^{J} \\
& =\sum_{\substack{i^{\prime}, B, J_{b_{1}}, \ldots, J_{b_{k}}}} \sum_{n, p} \operatorname{dim} \widetilde{H}^{n}\left(\Sigma K\left(L_{1}, \ldots, L_{m}\right)_{J}\right) \operatorname{dim} \widetilde{H}^{p}\left(\widehat{A}^{J_{b_{1}}} \wedge \cdots \wedge \widehat{A}^{J_{b_{k}}}\right) s^{i^{\prime}} \bar{t}^{J} \\
& =\sum_{\substack{i^{\prime}, B, J_{b_{1}}, \ldots, J_{b_{k}}}} \sum_{n, p}\left(\sum_{r, r_{1}, \ldots, r_{k}} \operatorname{dim} \widetilde{H}^{r}\left(\Sigma K_{B}\right) \prod_{i=1}^{k} \operatorname{dim} \widetilde{H}^{r_{i}}\left(\left.\Sigma L_{b_{i}}\right|_{J_{b_{i}}}\right)\right) \\
& \times\left(\sum_{c_{1}, \ldots, c_{k}} \prod_{i=1}^{k} \operatorname{dim} \widetilde{H}^{c_{i}}\left(\widehat{A}^{J_{i}}\right)\right) s^{i^{\prime}} \bar{t}^{J} \\
& =\sum_{\substack{i^{\prime}, B, J_{b_{1}}, \ldots, J_{b_{k}}}} \sum_{n, p} \sum_{r} \operatorname{dim} \widetilde{H}^{r}\left(\Sigma K_{B}\right) \\
& \times \prod_{i=1}^{k} \sum_{\substack{r_{1}, \ldots, r_{k}, c_{1}, \ldots, c_{k}}} \operatorname{dim} \widetilde{H}^{r_{i}+c_{j}}\left(\left.\Sigma L_{b_{i}}\right|_{J_{b_{i}}} \wedge \widehat{A}^{J_{b_{i}}}\right) s^{i^{\prime}} \bar{t}^{J_{b_{1}}} \cdots \bar{t}^{J_{b_{k}}}
\end{aligned}
$$

Next, we will use a change of variables in order to rewrite this in a recognizable form. Let

$$
r_{i}+c_{j}=a_{i+j}
$$

Then $i^{\prime}=n+p=r+\sum r_{i}+\sum c_{i}=r+\sum a_{i+j}=r+\sum a_{u}$. Therefore,

$$
\begin{aligned}
& \beta_{Z_{K\left(L_{1}, \ldots, L_{m}\right)}(\underline{C A}, \underline{A})}(s, \bar{t}) \\
& =\sum_{\substack{i^{\prime}, B, J_{b_{1}}, \ldots, J_{b_{k}}}} \sum_{n, p} \sum_{r} \operatorname{dim} \widetilde{H}^{r}\left(\Sigma K_{B}\right) \times \prod_{i=1}^{k} \sum_{\substack{r_{1}, \ldots, r_{k}, c_{1}, \ldots, c_{k}}} \operatorname{dim} \widetilde{H}^{r_{i}+c_{j}}\left(\left.\Sigma L_{b_{i}}\right|_{J_{b_{i}}} \wedge \widehat{A}^{J_{b_{i}}}\right) s^{i^{\prime}} \bar{t}^{J_{b_{1}}} \ldots \bar{t}^{J_{b_{k}}} \\
& =\sum_{B, r} \operatorname{dim} \widetilde{H}^{r}\left(\Sigma K_{B}\right) s^{r} \prod_{i=1}^{k} \sum_{J_{b}, a_{u}} \operatorname{dim} \widetilde{H}^{a_{u}}\left(\left.\Sigma L_{b_{i}}\right|_{J_{b_{i}}} \wedge \widehat{A}^{J_{b_{i}}}\right) s^{a_{u}} \bar{t}^{J_{s}} \\
& =\beta_{Z_{K}\left(D^{1}, S^{0}\right)}\left(s, \widetilde{\beta}_{Z_{L_{1}}(\underline{C A}, \underline{A})}(s, \bar{t}), \ldots, \widetilde{\beta}_{L_{m}(\underline{C A}, \underline{A})}(s, \bar{t})\right) .
\end{aligned}
$$


Example 5. Consider the composed simplicial complex from Example 2. We need to compute the reduced beta-polynomials of each complex $L_{i}$. Since the full subcomplex of $L_{1}$ associated to $\{11\}$ is the empty set,

$$
\widetilde{\beta}_{Z_{L_{1}}(\underline{C A}, \underline{A})}=\sum \operatorname{dim} \widetilde{H}^{i}\left(\Sigma \emptyset \wedge A_{11}\right) s^{i} t_{11} .
$$

The full subcomplexes of $L_{2}$ are all contractible, so its beta-polynomial is the zero polynomial. The only nontrivial full subcomplex of $L_{3}$ is associated to $\{31,32\}$, and hence its reduced beta-polynomial is

$$
\widetilde{\beta}_{Z_{L_{3}}(\underline{C A}, \underline{A})}=\sum \operatorname{dim} \widetilde{H}^{i}\left(\Sigma \partial \Delta^{1} \wedge A_{31} \wedge A_{32}\right) s^{i} t_{31} t_{32} .
$$

The noncontractible full subcomplexes of $K$ are $\{1,2\},\{1,3\},\{1,2,3\}$. Since the betapolynomial of $L_{2}$ is zero, any subsets of [3] that contain 2 do not contribute any nontrivial terms. Apply Proposition 4.8,

$$
\begin{aligned}
& \beta_{Z_{K\left(L_{1}, \ldots L_{m}\right)}(\underline{C A}, \underline{A})}(s, \bar{t})=\beta_{Z_{K}\left(D^{1}, S^{0}\right)}\left(s, \widetilde{\beta}_{Z_{L_{1}}(\underline{C A}, \underline{A})}(s, \bar{t}), \ldots, \widetilde{\beta}_{Z_{L_{m}}(\underline{C A}, \underline{A})}(s, \bar{t})\right) \\
& \quad=\sum_{i \in \mathbb{Z}, J \subset[m]} \operatorname{dim} \widetilde{H}^{i}\left(\sum \mathcal{K}_{J}\right) s^{i}\left(\widetilde{\beta}_{Z_{\underline{L}}(\underline{C A}, \underline{A})}\right)^{J} \\
& \quad=1+\sum \operatorname{dim} H^{i}\left(\sum \partial \Delta^{1}\right) s^{i}\left(\widetilde{\beta}_{Z_{L_{1}}(\underline{C A}, \underline{A})}\right)\left(\widetilde{\beta}_{Z_{L_{3}}(\underline{C A}, \underline{A})}\right) \\
& \quad=1+s\left(\sum \operatorname{dim} \widetilde{H}^{i}\left(\sum \emptyset \wedge A_{11}\right) s^{i} t_{11}\right)\left(\sum \operatorname{dim} \widetilde{H}^{i}\left(\Sigma \partial \Delta^{1} \wedge A_{31} \wedge A_{32}\right) s^{i} t_{31} t_{32}\right) .
\end{aligned}
$$

Since all full subcomplexes of $K\left(L_{1}, L_{2}, L_{3}\right)$ are contractible except those associated to the empty set and the set $\{11,31,32\}$, its beta-polynomial is

$$
\begin{aligned}
\beta_{Z_{K\left(L_{1}, L_{2}, L_{3}\right)}(\underline{C A}, \underline{A})}(s, \bar{t}) & =\sum_{i \in \mathbb{Z}, J} \operatorname{dim} \widetilde{H}^{i}\left(\Sigma \mathcal{K}_{J} \wedge \widehat{A}^{J}\right) s^{i} \bar{t}^{J} \\
& =1+\sum \operatorname{dim} \widetilde{H}^{i}\left(\Sigma \partial \Delta^{2} \wedge A_{11} \wedge A_{31} \wedge A_{32}\right) s^{i} t_{11} t_{31} t_{32} .
\end{aligned}
$$

If for example $A_{i}=S^{2}$ for all $i$, then both expressions 4.5 and 4.4 simplify to

$$
1+s^{8} t_{11} t_{31} t_{32} \text {. }
$$

\section{2. $\quad$ Ring structure}

Recall the generalized Stanley-Reisner ideal $I(K)$ in $\widetilde{H}^{*}\left(X_{1}\right) \otimes \cdots \otimes \widetilde{H}^{*}\left(X_{m}\right)$,

$$
I(K)=\left\langle x_{i_{1}} \otimes \cdots \otimes x_{i_{k}} \mid\left\{i_{1}, \ldots, i_{k}\right\} \notin K\right\rangle .
$$

Since the generalized Stanley-Reisner ideal is essential to understanding the ring structure of $H^{*}\left(Z_{K}(\underline{X}, \underline{A})\right)$, we will describe the generalized Stanley-Reisner ideal in the case that the underlying simplicial complex is a composition $K\left(L_{1}, \ldots, L_{m}\right)$ in terms of the generalized Stanley-Reisner ideal of $K, L_{1}, \ldots, L_{m}$.

Proposition 4.9. The generalized Stanley-Reisner ideal of $K\left(L_{1}, \ldots, L_{m}\right)$ satisfies

$$
I\left(K\left(L_{1}, \ldots, L_{m}\right)\right)=\left\langle c_{i_{1}} \otimes \cdots \otimes c_{i_{k}} \mid\left\{i_{1}, \ldots, i_{k}\right\} \notin K\right\rangle,
$$

where $c_{i} \in I\left(L_{i}\right)$ for $i_{1} \leqslant i \leqslant i_{k}$. 
Proof. Recall from the proof of Theorem 4.1 that $C_{i}=I\left(L_{i}\right)$.

Suppose $c^{\sigma} \in I\left(K\left(L_{1}, \ldots, L_{m}\right)\right)$ where $\sigma \subset\left[\sum l_{i}\right]$ is such that $\sigma \notin K\left(L_{1}, \ldots, L_{m}\right)$. Recall from the equivalent definition of $K\left(L_{1}, \ldots L_{m}\right.$ ) (after Definition 2.6) that $\sigma$ is of the form

$$
\sigma=\bigcup_{i \in[m]} \sigma_{i}
$$

where $\sigma_{i} \subset\left[l_{i}\right]$ and $\left\{i \in[m] \mid \sigma_{i} \notin L_{i}\right\} \notin K$. Let $A=\left\{i \in[m] \mid \sigma_{i} \notin L_{i}\right\}=\left\{i_{1}, \ldots, i_{k}\right\}$. In other words $c^{\sigma}=c^{A}=c_{i_{1}} \otimes \cdots \otimes c_{i_{k}}$ where $A \notin K$ and $c_{i} \in I\left(L_{i}\right)$. It follows that $c^{\sigma} \in\left\langle c_{i_{1}} \otimes \cdots \otimes c_{i_{k}} \mid\left\{i_{1}, \ldots, i_{k}\right\} \notin K\right\rangle$.

Suppose $c=c_{i_{1}} \otimes \cdots \otimes c_{i_{k}} \in\left\langle c_{i_{1}} \otimes \cdots \otimes c_{i_{k}} \mid\left\{i_{1}, \ldots, i_{k}\right\} \notin K\right\rangle$ where $c_{i} \in I\left(L_{i}\right)$ for $i_{1} \leqslant i \leqslant i_{k}$. Since $c_{i} \in I\left(L_{i}\right)$, it is of the form $c_{i}=c^{\tau_{i}}$ for some $\tau_{i} \notin L_{i}$. Since $\left\{i_{1}, \ldots, i_{k}\right\}$ $\notin K$, we have that $\tau=\cup \tau_{i} \notin K\left(L_{1}, \ldots, L_{m}\right)$. Therefore, $c=c^{\tau} \in I\left(K\left(L_{1}, \ldots, L_{m}\right)\right)$.

Example 6. A minimal nonface of $K$ is a set that is not a simplex of $K$ but every subset of it is a simplex of $K$. The generalized Stanley-Reisner ideal is generated by the minimal nonfaces. Suppose we have the following simplicial complexes:

$$
\begin{array}{lll}
L_{1} \subset[2], & L_{1}=\{\{11\},\{12\}\}, & I\left(L_{1}\right)=\left\langle c_{11} \otimes c_{12}\right\rangle, \\
L_{2} \subset[2], & L_{2}=\{\{21\}\}, & I\left(L_{2}\right)=\left\langle c_{22}\right\rangle, \\
K \subset[2], & K=\{\{2\}\}, & I(K)=\left\langle c_{1}\right\rangle .
\end{array}
$$

The composition $K\left(L_{1}, L_{2}\right)=L_{1} * \Delta^{1}=\{\{11,21,22\},\{12,21,22\}\}$ and hence

$$
I\left(K\left(L_{1}, L_{2}\right)\right)=\left\langle c_{11} \otimes c_{12}\right\rangle .
$$

\section{The pair $\left(L_{i}, \emptyset\right)$}

In this section we will find a formula for the cohomology groups of $Z_{Z_{K}^{*}\left(L_{i}, \emptyset\right)}(\underline{X}, \underline{A})$, the polyhedral product over a polyhedral join given by the pairs $\left(L_{i}, \emptyset\right)$. In this case, we get a similar formula to Theorem 2.7

$$
Z_{Z_{K}^{*}\left(L_{i}, \emptyset\right)}(\underline{X}, \underline{A})=Z_{K}\left(Z_{L_{i}}(\underline{X}, \underline{A}), \prod_{j \in\left[l_{i}\right]} A_{j}\right) .
$$

As an application, we can write the polyhedral product $Z_{K}\left(S^{n}, \vee S^{0}\right)$ as the real moment-angle complex $Z_{Z_{K}^{*}\left(\partial \Delta^{\left.n_{i}, \emptyset\right)}\right.}\left(D^{1}, S^{0}\right)$.

It follows from the discussion in Section 3 that the kernel, cokernel and image can be computed if the links of simplices in $L_{i}$ can be described in general. Note that $L_{i}$ and its subsimplicial complex, $\emptyset$, do not have any (nontrivial) simplices in common, so the links do not present any issues. Equation (5.1) and Theorem 2.13 imply the following formula.

Theorem 5.1. Given simplicial complexes $L_{i}$ on the vertex sets $\left[l_{i}\right]$ with no ghost vertices and pairs $\left(X_{i j}, A_{i j}\right)$, where $i$ varies in $[m]$ and $j$ varies in $\left[l_{i}\right]$, that satisfy 
the freeness condition of Definition 2.11 with decompositions

$$
\begin{aligned}
& H^{*}\left(X_{i j}\right)=B_{i j} \oplus C_{i j}, \\
& H^{*}\left(A_{i j}\right)=B_{i j} \oplus E_{i j} .
\end{aligned}
$$

Then we have

$$
\begin{aligned}
& H^{*}\left(Z_{Z_{K}^{*}\left(L_{i}, \emptyset\right)}(\underline{X}, \underline{A})\right) \\
& \quad=\bigoplus_{J, \tau, I, \sigma} E^{T} \otimes B^{(T \cup S)^{c}} \otimes C^{S} \otimes\left(\bigotimes_{v \in \tau} \widetilde{H}^{*}\left(\Sigma|l k(\sigma)|_{I}\right)\right) \otimes \widetilde{H}^{*}\left(\Sigma|l k(\tau)|_{J^{c}}\right),
\end{aligned}
$$

where

1. $J \subset[m]$ with a simplex $\tau$ of $K$ such that $\tau \subset J$;

2. For $v \in \tau$, take subsets $I_{v} \subset\left[l_{v}\right]$ and a simplex $\sigma \in L_{v}$ such that $\sigma \subset I^{c}$. For $k \in[m] \backslash J$, consider subsets $I_{k} \subset\left[l_{k}\right]$. Then $T$ and $S$ are defined by

$$
T=\left(\bigcup_{[m] \backslash J} I_{k}\right) \cup\left(\bigcup_{v \in \tau} I_{v}\right), \quad S=\bigcup_{v \in \tau} \sigma_{v} .
$$

Proof. From Definition 2.11, we need to find the kernel, $E_{i}$, image, $B_{i}$, and cokernel, $C_{i}$, of the map

$$
H^{*}\left(Z_{L_{i}}(\underline{X}, \underline{A})\right) \rightarrow H^{*}\left(\prod_{j \in\left[l_{i}\right]} A_{j}\right)
$$

where $H^{*}\left(\prod_{j \in\left[l_{i}\right]} A_{j}\right)=E_{i} \oplus B_{i}$ and $H^{*}\left(Z_{L_{i}}(\underline{X}, \underline{A})\right)=C_{i} \oplus B_{i}$. Since the cohomology of each space in the pair is given by

$$
\begin{aligned}
H^{*}\left(\prod_{j \in\left[l_{i}\right]} A_{j}\right)= & \bigoplus_{I \subset\left[l_{i}\right]} B^{I} \otimes E^{I^{c}} \\
H^{*}\left(Z_{L_{i}}(\underline{X}, \underline{A})\right)= & \bigoplus_{\substack{\sigma \subset I \subset[m] \\
\sigma \in L_{i}}} E^{I^{c}} \otimes \widetilde{H}^{*}\left(\Sigma|l k(\sigma)|_{I^{c}}\right) \otimes Y^{I, \sigma} \\
= & \left(\bigoplus_{I \subset[m]} E^{I^{c}} \otimes B^{I} \otimes \widetilde{H}^{*}\left(\left.\Sigma \mathcal{L}_{i}\right|_{I^{c}}\right)\right) \\
& \left.\oplus \bigoplus_{\substack{\sigma \subset I \subset[m] \\
\emptyset \neq \sigma \in L_{i}}} E^{I^{c}} \otimes \widetilde{H}^{*}\left(\Sigma|l k(\sigma)|_{I^{c}}\right) \otimes Y^{I, \sigma}\right)
\end{aligned}
$$

and the full subcomplex $\left.\mathcal{L}_{i}\right|_{I^{c}}$ is only empty when $I^{c}=\emptyset$ (because $L_{i}$ has no ghost vertices), we have that

$$
\begin{aligned}
B_{i} & =B^{\left[l_{i}\right]} \\
E_{i} & =\bigoplus_{I \subsetneq\left[l_{i}\right]} B^{I} \otimes E^{I^{c}}, \\
C_{i} & =\bigoplus_{\substack{\sigma \subset I \subsetneq[m] \\
\sigma \in L_{i} \\
\emptyset \neq \sigma \in L_{i}}} E^{I^{c}} \otimes \widetilde{H}^{*}\left(\Sigma|l k(\sigma)|_{I^{c}}\right) \otimes Y^{(I, \sigma)},
\end{aligned}
$$


where " $\sigma, I \neq \emptyset "$ means that $\sigma$ and $I$ are not both the empty set. Then substituting,

$$
\begin{aligned}
H^{*}\left(Z_{Z_{K}^{*}\left(L_{i}, \emptyset\right)}(\underline{X}, \underline{A})\right) & =\bigoplus_{J, \tau} \widetilde{H}^{*}\left(\Sigma|l k(\tau)|_{J^{c}}\right) \otimes E^{[m] \backslash J} \otimes C^{\tau} \otimes B^{J \backslash \tau} \\
= & \bigoplus_{J, \tau}\left(\widetilde{H}^{*}\left(\Sigma|l k(\tau)|_{J^{c}}\right) \otimes \bigotimes_{k \in[m] \backslash J}\left(\bigoplus_{L \subset\left[l_{k}\right]} E^{L} \otimes B^{L^{c}}\right)\right. \\
& \left.\otimes \bigotimes_{v \in \tau}\left(\bigoplus_{\substack{I \subset\left[l_{v}\right], \sigma \in L_{v}, \sigma, I \neq \emptyset}} E^{I} \otimes Y^{I^{c}, \sigma} \otimes \widetilde{H}^{*}\left(\Sigma|l k(\sigma)|_{I}\right)\right) \otimes \bigotimes_{u \in J \backslash \tau} B^{\left[l_{u}\right]}\right) \\
= & \bigoplus_{J, \tau,}\left(\widetilde{H}^{*}\left(\Sigma|l k(\tau)|_{J^{c}}\right) \otimes \bigotimes_{k \in[m] \backslash J}\left(E^{L} \otimes B^{L^{c}}\right)\right. \\
& \left.\otimes \bigotimes_{v \in \tau}\left(E^{I} \otimes Y^{I^{c}, \sigma} \otimes \widetilde{H}^{*}\left(\Sigma|l k(\sigma)|_{I}\right)\right) \otimes \bigotimes_{u \in J \backslash \tau} B^{\left[l_{u}\right]}\right) .
\end{aligned}
$$

Corollary 5.2. Suppose $\left(X_{i j}, A_{i j}\right)=\left(C A_{i j}, A_{i j}\right)$. Then

$$
\begin{aligned}
H^{*}\left(Z_{Z_{K}^{*}\left(L_{i}, \emptyset\right)}(\underline{C A}, \underline{A})\right) \\
=\bigoplus_{J, \tau, I_{k}, I_{v} \neq \emptyset}\left(\widetilde{H}^{*}\left(\widehat{A}^{T}\right) \otimes\left(\bigotimes_{v \in \tau} \widetilde{H}^{*}\left(\left.\Sigma \mathcal{L}_{v}\right|_{I_{v}}\right)\right) \otimes \widetilde{H}^{*}\left(\Sigma|l k(\tau)|_{J^{c}}\right)\right),
\end{aligned}
$$

where

1. $J \subset[m]$ with a simplex $\tau$ of $K$ such that $\tau \subset J$;

2. For $k \in[m] \backslash J$ and $v \in \tau$, take subsets $I_{v} \subset\left[l_{v}\right]$ and $I_{k} \subset\left[l_{k}\right]$, and define $T$ :

$$
T:=\left(\bigcup_{k \in[m] \backslash J} I_{k}\right) \cup\left(\bigcup_{v \in \tau} I_{v}\right) .
$$

Proof. With the given pairs, we know that $C_{i j}=0, B_{i j}=1$ and $E_{i j}=\widetilde{H}^{*}\left(A_{i j}\right)$ for all $i j$. Since $C_{i j}=0, \sigma$ in Proposition 5.1 must be the empty set. Therefore $I$ cannot be the empty set and

$$
\begin{aligned}
H^{*}\left(Z_{Z_{K}^{*}\left(L_{i}, \emptyset\right)}(\underline{C A}, \underline{A})\right) & \\
= & \bigoplus_{\substack{J, \tau, I_{k}, I_{v} \neq \emptyset}}\left(\left(\bigotimes_{k \in[m] \backslash J} E^{I_{k}} \otimes B^{I_{k}^{c}}\right) \otimes\left(\bigotimes_{v \in \tau} E^{I_{\alpha}} \otimes B^{I_{v}^{c}} \otimes \widetilde{H}^{*}\left(\left.\Sigma L_{v}\right|_{I_{v}}\right)\right)\right. \\
& \left.\otimes\left(\bigotimes_{j \in J \backslash \tau} B^{\left[l_{\beta}\right]}\right) \otimes \widetilde{H}^{*}\left(\Sigma|l k(\tau)|_{J^{c}}\right)\right) \\
= & \bigoplus_{J, \tau, I_{k}, I_{\alpha} \neq \emptyset}\left(\left(\bigotimes_{k \in[m] \backslash J} E^{I_{k}}\right) \otimes\left(\bigotimes_{v \in \tau} E^{I_{v}} \otimes \widetilde{H}^{*}\left(\left.\Sigma L_{v}\right|_{I_{v}}\right)\right) \otimes \widetilde{H}^{*}\left(\Sigma|l k(\tau)|_{J^{c}}\right)\right) .
\end{aligned}
$$




\section{References}

[1] A. Ayzenberg, Composition of simplicial complexes, polytopes and multigraded Betti numbers, Trans. Moscow Math. Soc. 74 (2013), no. 2, 175-202. MR 3235795.

[2] A. Bahri, M. Bendersky, F.R. Cohen, and S. Gitler, The polyhedral product functor: a method of decomposition for moment-angle complexes, arrangements and related spaces, Adv. Math. 225 (2010), no. 3, 1634-1668. MR 2673742 (2012b:13053).

[3] A. Bahri, M. Bendersky, F.R. Cohen, and S. Gitler, Operations on polyhedral products and a new topological construction of infinite families of toric manifolds, Homology Homotopy Appl. 17 (2015), no. 2, 137-160. MR 3426378.

[4] A. Bahri, M. Bendersky, F.R. Cohen, and S. Gitler, A spectral sequence for polyhedral products, Adv. Math. 308 (2017), 767-814, MR 3600073.

[5] V.M. Buchstaber and T.E. Panov, Torus Actions and Their Applications in Topology and Combinatorics, Univ. Lecture Ser., vol. 24, American Mathematical Society, Providence, RI, 2002. MR 1897064 (2003e:57039).

[6] S. Choi and H. Park, Wedge operations and torus symmetries, Tohoku Math. J. (2) 68 (2016), no. 1, 91-138, MR 3476138.

[7] G. Ewald, Spherical complexes and nonprojective toric varieties, Discrete Comput. Geom. 1 (1986), no. 2, 115-122, MR 0834053 (87i:52012).

[8] S. Gitler and S. López de Medrano, Intersections of quadrics, moment-angle manifolds and connected sums, Geom. Topol. 17 (2013), no. 3, 1497-1534. MR 3073929.

[9] J. Grbić and S. Theriault, The homotopy type of the polyhedral product for shifted complexes, Adv. Math. 245 (2013), 690-715. MR 3084441.

[10] I. Limonchenko, Families of minimally non-Golod complexes and polyhedral products, Dal?nevost. Mat. Zh., 15 (2015), no. 2, 222-237, MR 3593998.

[11] Yu.M. Ustinovskii, Toral rank conjecture for moment-angle complexes, Math. Notes 90 (2011), no. 1, 279, MR 2918445.

[12] Q. Zheng, The complement of polyhedral product spaces and the dual simplicial complexes, J. Pure Appl. Algebra 222 (2018), no. 9, 2470-2489, MR 3783000.

Elizabeth Vidaurre elizabeth.vidaurre@rochester.edu

Mathematics Department, University of Rochester, 500 Wilson Blvd. Rochester, NY 14627, USA 